\title{
The Poisson-G Family of Distributions with Applications
}

T. H. M. Abouelmagd

Management Information System Department, Taibah

University, Saudi Arabia

Department of Statistics, Mathematics and Insurance

Benha University, Egypt

tabouelmagd@taibahu.edu.sa

M. S. Hamed

Management Information System Department, Taibah

University, Saudi Arabia

Department of Statistics, Mathematics and Insurance

Benha University, Egypt

moswilem@gemail.com

Abd El Hadi N. Ebraheim

Institute of Statistical Studies \& Research

Cairo University, Egypt

dr.hadi@cu.edu.eg

\begin{abstract}
We define and study a new class of continuous distributions called the Poisson-G family. We present three of its several special models. Some of its mathematical properties including explicit expressions for the ordinary and incomplete moments, quantile and generating functions and entropies are provided. The estimations of the model parameters is carried out using maximum likelihood method. The flexibility of the new family is illustrated by means of two applications to real data sets.
\end{abstract}

Keywords: Entropy, Generating Function, Generalized Exponential Poisson, Maximum Likelihood.

\section{Introduction}

Recently, Many generalized families of distributions have been proposed and extensively used in modeling data in various applied sciences such as economics, finance, insurance, engineering and life testing. However, there is a clear need for extended forms of these distributions by adding one or more shape parameter(s) in order to obtain greater flexibility in modelling these data.

There are many well-known families in the literature. For example, the exponentiated-G (Exp-G) (Gupta et al., 1998), beta-G (Eugene et al., 2002), transmuted-G (Shaw and Buckley, 2007), Kumaraswamy-G (Cordeiro and de Castro, 2011), McDonald-G 
(Alexander et al., 2012), transmuted exponentiated generalized-G (Yousof et al., 2015) and generalized transmuted-G (Nofal et al., 2017) families.

In this paper, we propose and study the Poisson-G (Po-G) family of distributions. The main advantage of the Po- $G$ family relies on the fact that practitioners will have a quite flexible one-parameter class to fit real life data in applied fields. It may serve as a good alternative to other one, two or three-parameter families. It also may work better, in terms of model fitting, than other classes of distributions in certain practical situations which cannot always be guaranteed. Furthermore, a comprehensive account of some of its mathematical properties are provided. We prove empirically that the special models of the Po- $G$ family can provide better fit than other competitive models generated by the above mentioned classes.

Let $p(t)$ be the probability density function (pdf) of a random variable $T \in a, b$ ] for $-\infty<a<b<\infty$ and let $W[G(x)]$ be a function of the cumulative distribution function (cdf) of a random variable $X$ such that $W[G(x)]$ satisfies the following conditions:

(i) $W[G(x)] \in a, b]$,

(ii) $W[G(x)]$ is differentiable and monotonically non - decreasing, and

(iii) $W[G(x)] \rightarrow a$ as $x \rightarrow-\infty$ and $W[G(x)] \rightarrow b$ as $x \rightarrow \infty$.

Recently, Alzaatreh et al. (2013) proposed the T-X family with cdf

$$
F(x)=\int_{a}^{W[G(x)]} p(t) d t
$$

where $W[G(x)]$ satisfies the conditions (1). The corresponding pdf of (2) is defined by

$$
f(x)=\left\{\frac{d}{d x} W[G(x)]\right\} p\{W[G(x)]\} .
$$

Based on generalized exponential power series distribution (Mahmoudi and Jafari, 2012), first we define the Po- $G$ family of distributions.

For $W[G(x)]=-\log [1-G(x ; \xi)]$ and $p(t)$ be the pdf of generalized exponential Poisson with $\alpha=\beta=1$, we define the cdf of the new Po- $G$ family of distributions by

$$
\begin{aligned}
F(x ; \theta, \xi) & =\int_{0}^{-\log [1-G(x ; \xi)]} \frac{\theta \exp (-t) \exp \left[\theta\left(1-e^{-t}\right)\right]}{e^{\theta}-1} d t \\
& =\frac{\exp [\theta G(x ; \xi)]-1}{e^{\theta}-1},
\end{aligned}
$$

where $G(x ; \xi)$ is the baseline cdf depending on a parameter vector $\xi$ and $\theta>0$ is a shape parameter.

The corresponding pdf of (4) is given by

$$
f(x ; \theta, \xi)=\frac{\theta g(x ; \xi)}{e^{\theta}-1} \exp [\theta G(x ; \xi)]
$$

We denote a random variable having density function (5) by $X \sim \operatorname{Po}-G(\theta, \xi)$. The reliability function (rf), hazard rate function (hrf) and cumulative hazard rate function (chrf) of $X$ are, respectively, given by

$$
R(x ; \theta, \xi)=\frac{\exp (\theta)-\exp [\theta G(x ; \xi)]}{e^{\theta}-1},
$$


and

$$
h(x ; \theta, \xi)=\frac{\theta g(\xi \xi) \exp [\theta G(x ; \xi)]}{\exp (\theta)-\exp [\theta G(x ; \xi)]}
$$

$$
H(x ; \theta, \xi)=-\ln \left\{\frac{\exp (\theta)-\exp [\theta G(x ; \xi)]}{e^{\theta}-1}\right\}
$$

Henceforth, we will omit the dependence on the model parameters for simplicity and write $g(x)=g(x ; \xi), F(x)=F(x ; \theta, \xi)$ and $f(x)=f(x ; \theta, \xi)$, etc.

The rest of the paper is outlined as follows. In Section 2, we derive a very useful representation for the Po- $G$ density function. Three special models of this family are presented in Section 3 and some plots of their pdfs and hrfs are given. We obtain in Section 4 some general mathematical properties of the proposed family including ordinary and incomplete moments, quantile function (qf), moment generating function (mgf) and entropies. Maximum likelihood estimation of the model parameters is investigated in Section 5. In Section 6, simulation results to assess the performance of the proposed maximum likelihood estimation procedure are discussed for one special model. In Section 7, we perform two applications to real data sets to illustrate the potentiality of three special models of the proposed family. Finally, some concluding remarks are presented in Section 8.

\section{Linear representation}

In this section, we provide a useful mixture representation for the Po- $G$ family in terms of Exp- $G$ densities. The pdf (5) can be expressed as

$$
f(x)=\frac{\theta g(x)}{e^{\theta}-1} \exp [\theta G(x)]
$$

Using the power series for $\exp [\theta G(x)]$, the pdf of the Po- $G$ family can be expressed as

$$
f(x)=\frac{1}{e^{\theta}-1} \sum_{k=0}^{\infty} \frac{\theta^{k+1}}{(k+1) !} h_{k+1}(x) .
$$

Then, we can write the pdf of the Po- $G$ family as

$$
f(x)=\sum_{k=0}^{\infty} \varphi_{k} h_{k+1}(x) .
$$

where $\varphi_{k}=\theta^{k+1} /\left[\left(e^{\theta}-1\right)(k+1) !\right]$ and $h_{\alpha}(x)=\alpha g(x) G(x)^{\alpha-1}$ is the Exp-G density function with power parameter $\alpha$.

Equation (6) reveals that the density function of $X$ is a mixture of the baseline density and the $\operatorname{Exp}-G$ density with power parameter $k+1$. Thus, some structural properties of the Po- $G$ family such as ordinary and incomplete moments, mean deviations and generating function can be determined from those properties of the Exp- $G$ distributions.

\section{Special models}


In this section, we provide three special models of the Po- $G$ family. The pdf (5) will be most tractable when $G(x ; \xi)$ and $g(x ; \xi)$ have simple analytic expressions. These special models generalize some well-known distributions corresponding to the baseline Weibull $(\mathrm{W})$, additive Weibull (AW) and Burr X (BX) distributions.

\subsection{The PoW Model}

Consider the cdf and pdf (for $x>0$ ) $G(x)=1-\exp \left[-(\alpha x)^{\beta}\right]$ and $g(x)=$ $\beta \alpha^{\beta} x^{\beta-1} \exp \left[-(\alpha x)^{\beta}\right]$, respectively, of the $\mathrm{W}$ distribution with positive parameters $\alpha$ and $\beta$. Then, the pdf and cdf of the PoW model are, respectively, given by

and

$$
f(x)=\frac{\theta \beta \alpha^{\beta} x^{\beta-1} \exp \left[-(\alpha x)^{\beta}\right]}{e^{\theta-1}} \exp \left\{\theta-\theta \exp \left[-(\alpha x)^{\beta}\right]\right\}
$$

$$
F(x)=\frac{\exp \left\{\theta-\theta \exp \left[-(\alpha x)^{\beta}\right]\right\}-1}{e^{\theta}-1}
$$

For $\beta=2$, we have the Po-Rayleigh (PoR) distribution. For $\beta=1$, it gives the Poexponential (PoE) model. Plots of the PoW density and hrf for selected parameter values are displayed in Figure 1.

\subsection{The PoAW Model}

The AW distribution with parameters $\alpha, \beta, \gamma, \delta \geq 0$ where $\delta<1<\beta$ (or $\beta<1<\delta$ ), $\delta$ and $\beta$ are the shape parameters and $\alpha$ and $\gamma$ are scale parameters, has pdf and cdf (for $x>0)$ given by $g(x)=\left(\alpha \delta x^{\delta-1}+\gamma \beta x^{\beta-1}\right) \exp \left(-\alpha x^{\delta}-\gamma x^{\beta}\right)$ and $G(x)=1-$ $\exp \left(-\alpha x^{\delta}-\gamma x^{\beta}\right)$, respectively. Then, the pdf and cdf of the PoAW model are given by

and

$$
f(x)=\frac{\theta\left(\alpha \delta x^{\delta-1}+\gamma \beta x^{\beta-1}\right)}{\left(e^{\theta}-1\right) \exp \left(\alpha x^{\delta}+\gamma x^{\beta}\right)} \exp \left[\theta-\theta \exp \left(-\alpha x^{\delta}-\gamma x^{\beta}\right)\right]
$$

$$
F(x)=\frac{\exp \left[\theta-\theta \exp \left(-\alpha x^{\delta}-\gamma x^{\beta}\right)\right]-1}{e^{\theta}-1}
$$

respectively. Figure 2 displays plots of the PoAW density and its hrf for selected parameter values.

\subsection{The PoBX Model}

The BX (also known as the generalized Raleigh) model with positive parameters $\alpha$ and $\beta$ has cdf and pdf (for $x>0$ ) given by $G(x)=\left\{1-\exp \left[-(\beta x)^{2}\right]\right\}^{\alpha}$ and $g(x)=$ $2 \alpha \beta^{2} x \exp \left[-(\beta x)^{2}\right]\left\{1-\exp \left[-(\beta x)^{2}\right]\right\}^{\alpha-1}$, respectively. Then, the PoBX pdf and cdf are given, respectively, by

and

$$
f(x)=\frac{2 \theta \alpha \beta^{2} x \exp \left[-(\beta x)^{2}\right]}{e^{\theta}-1}\left\{1-\exp \left[-(\beta x)^{2}\right]\right\}^{\alpha-1} \exp \left(\theta\left\{1-\exp \left[-(\beta x)^{2}\right]\right\}^{\alpha}\right)
$$

$$
F(x)=\frac{\exp \left\{\theta\left\{1-\exp \left[-(\beta x)^{2}\right]\right\}^{\alpha}\right\}-1}{\left(e^{\theta}-1\right)}
$$


Plots of the PoBX density and its hrf for selected parameter values are displayed in Figure 3 .
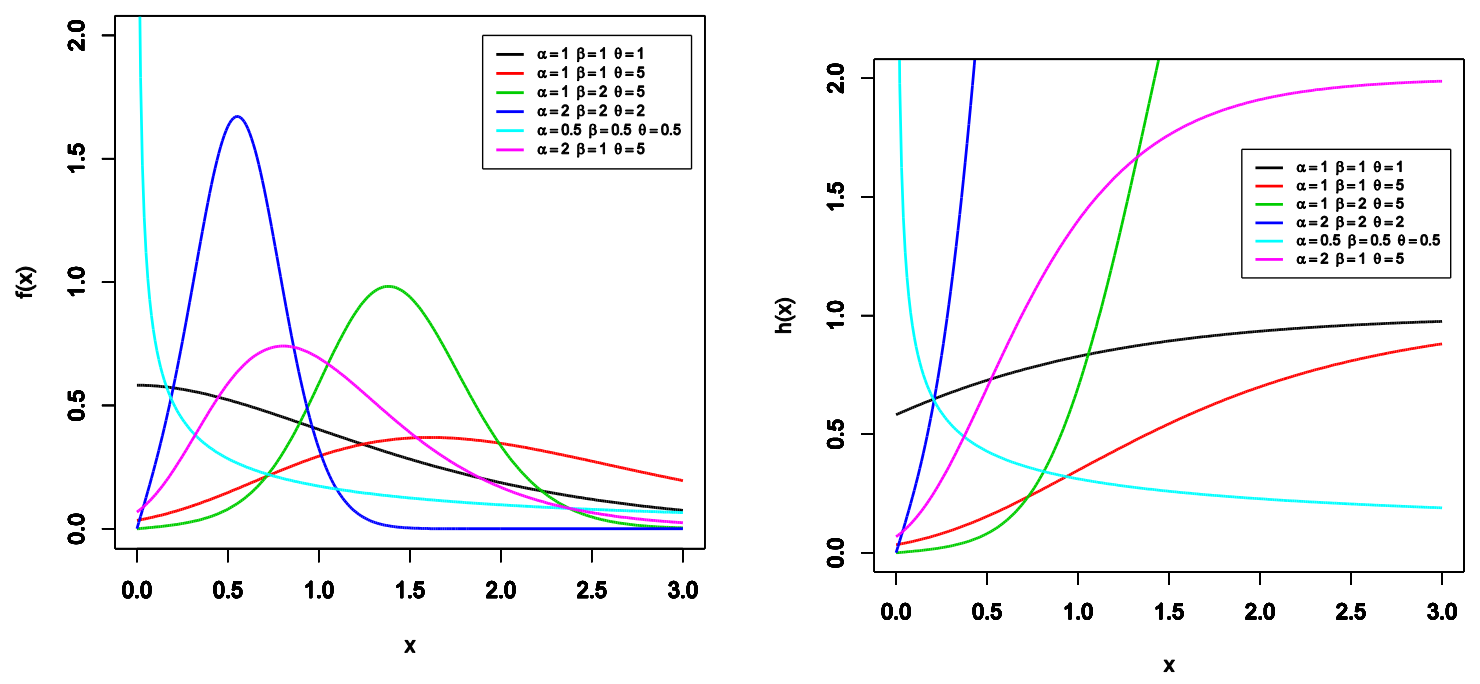

Figure 1: (a) pdf of PoW distribution and (b) hrf of PoW distribution
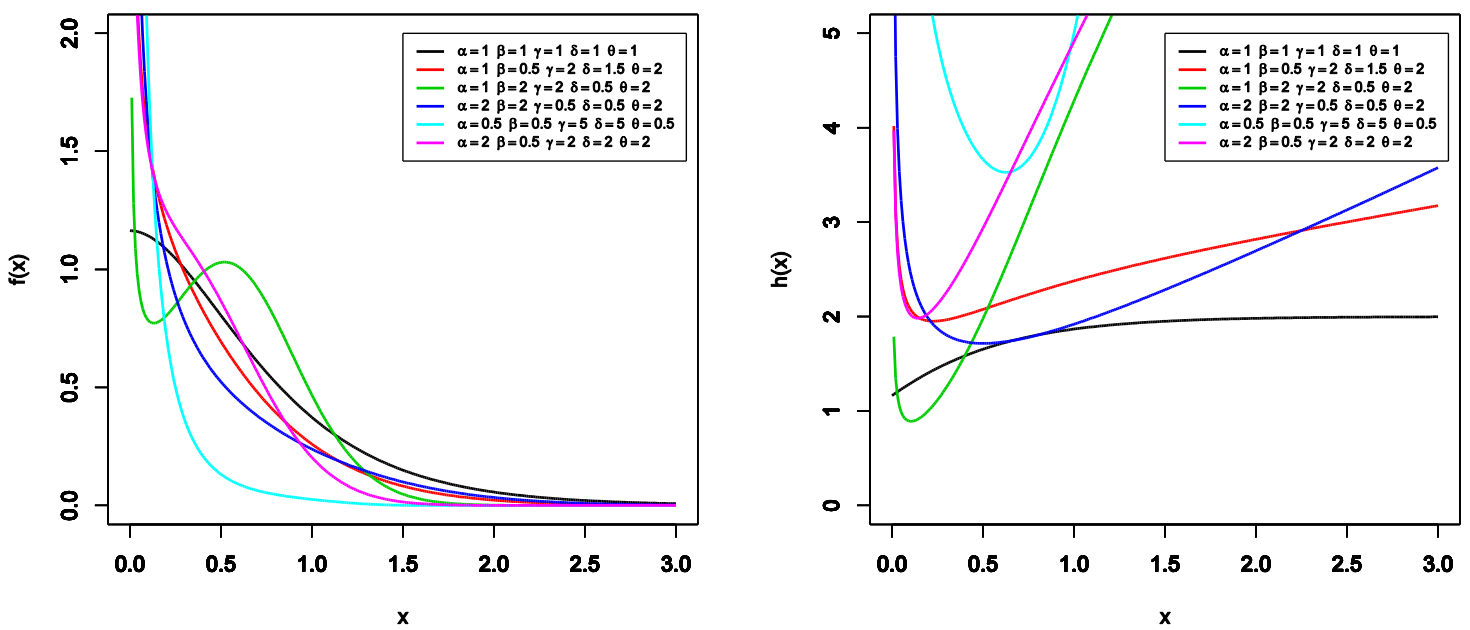

Figure 2: (a) pdf of PoAW distribution and (b) hrf of PoAW distribution

\section{Properties}

In this section, we derive some mathematical properties of the proposed family based on the linear representation derived in Section 2.

\subsection{Ordinary and incomplete moments}


The $r$ th moment of $X$, say $\mu_{r}^{\prime}$, follows from (6) as

$$
\mu_{r}^{\prime}=E\left(X^{r}\right)=\sum_{k=0}^{\infty} \varphi_{k} E\left(Y_{k+1}^{r}\right)
$$
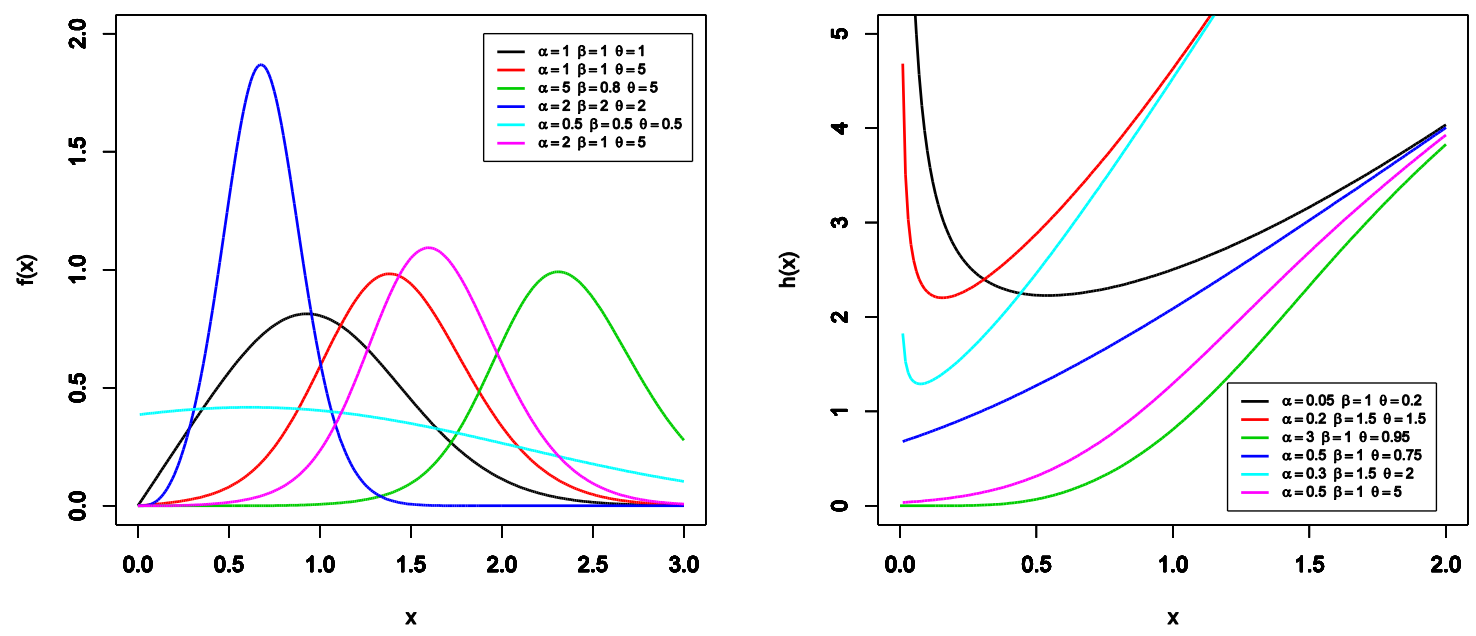

Figure 3: (a) pdf of PoBX distribution and (b) hrf of PoBX distribution

Henceforth, $Y_{k+1}$ denotes the Exp- $G$ distribution with power parameter $k+1$.

The $s$ th incomplete moment, say $\varphi_{s}(t)$, of $X$ can be expressed from (6) as

$$
\varphi_{s}(t)=\int_{-\infty}^{t} x^{s} f(x) d x=\sum_{k=0}^{\infty} \varphi_{k} \int_{-\infty}^{t} x^{s} h_{k+1}(x) d x
$$

The mean deviations about the mean $\left[\delta_{1}=E\left(\left|X-\mu_{1}^{\prime}\right|\right)\right]$ and about the median $\left[\delta_{2}=\right.$ $E(|X-M|)]$ of $X$ are given by $\delta_{1}=2 \mu_{1}^{\prime} F\left(\mu_{1}^{\prime}\right)-2 \varphi_{1}\left(\mu_{1}^{\prime}\right)$ and $\delta_{2}=\mu_{1}^{\prime}-2 \varphi_{1}(M)$, respectively, where $\mu_{1}^{\prime}=E(X), M=\operatorname{Median}(X)=Q(0.5)$ is the median, $F\left(\mu_{1}^{\prime}\right)$ is easily calculated from (4) and $\varphi_{1}(t)$ is the first incomplete moment given by (7) with $s=$ 1.

Now, we provide two ways to determine $\delta_{1}$ and $\delta_{2}$. First, a general equation for $\varphi_{1}(t)$ can be derived from (6) as

$$
\varphi_{1}(t)=\sum_{k=0}^{\infty} \varphi_{k} J_{k+1}(x)
$$

where $J_{k+1}(x)=\int_{-\infty}^{t} x h_{k+1}(x) d x$ is the first incomplete moment of the Exp-G distribution.

A second general formula for $\varphi_{1}(t)$ is given by

$$
\varphi_{1}(t)=\sum_{k=0}^{\infty} \varphi_{k} v_{k}(t)
$$

where $v_{k}(t)=(k+1) \int_{0}^{G(t)} Q_{G}(u) u^{k} d u$ can be computed numerically. 
These equations for $\varphi_{1}(t)$ can be applied to construct Bonferroni and Lorenz curves defined for a given probability $\pi$ by $B(\pi)=\varphi_{1}(q) /\left(\pi \mu_{1}^{\prime}\right)$ and $L(\pi)=\varphi_{1}(q) / \mu_{1}^{\prime}$, respectively, where $\mu_{1}^{\prime}=E(X)$ and $q=Q(\pi)$ is the qf of $X$ at $\pi$.

\subsection{Quantile and generating functions}

The qf of $X$, where $X \sim \operatorname{Po}-G(\theta, \xi)$, is obtained by inverting (4) to obtain $Q(u)=F^{-1}, 0<$ $u<1$.

Simulating the Po random variable is straightforward. If $U$ is a uniform variate on the unit interval $(0,1)$, then the random variable $X=Q(U)$ follows (4).

For simulating from Po- $G$ if $u \sim U(0,1)$, then solution of nonlinear equation

$$
x_{u}=G^{-1}\left\{\frac{1}{\theta} \log \left[1+u\left(e^{\theta}-1\right)\right]\right\} .
$$

Now, we provide two formulae for the $\operatorname{mgf} M_{X}(t)=E\left(e^{t X}\right)$ of $X$. Clearly, the first one can be derived from equation (6) as

$$
M_{X}(t)=\sum_{k=0}^{\infty} \varphi_{k} M_{k+1}(t)
$$

where $M_{k+1}(t)$ is the mgf of $Y_{k+1}$. Hence, $M_{X}(t)$ can be determined from the Exp-G generating function.

A second formula for $M_{X}(t)$ follows from (6) as

$$
M_{X}(t)=\sum_{k=0}^{\infty} \varphi_{k} \tau(t, k)
$$

where $\tau(t, k)=\int_{0}^{1} \exp \left[t Q_{H}(u)\right] u^{k} d u$ and $Q_{H}(u)$ is the qf corresponding to $H(x ; \phi)$, i.e., $Q_{H}(u)=H^{-1}(u ; \phi)$.

\subsection{Entropies}

The Rényi entropy of a random variable $X$ represents a measure of variation of the uncertainty. The Rényi entropy is defined by

$$
I_{\gamma}(X)=\frac{1}{1-\gamma} \log \int_{-\infty}^{\infty} f(x)^{\gamma} d x, \gamma>0 \text { and } \gamma \neq 1
$$

Using the pdf (5), we can write

$$
f(x)^{\gamma}=\left(\frac{\theta}{e^{\theta}-1}\right)^{\gamma} g(x ; \xi)^{\gamma} \exp [\theta \gamma G(x ; \xi)] .
$$

Using the power series for $\exp [\theta \gamma G(x ; \xi)]$, we an write

$$
f(x)^{\gamma}=\sum_{k=0}^{\infty} m_{k+1} h_{k+1}(x),
$$

where $m_{k+1}=\left[\theta /\left(e^{\theta}-1\right)\right]^{\gamma}(\theta \gamma)^{k} /(k+1)$ !.

Then, the Rényi entropy of the Po- $G$ family is given by 


$$
I_{\gamma}(X)=\frac{1}{1-\gamma} \log \left[\sum_{k=0}^{\infty} m_{k+1} \int_{-\infty}^{\infty} h_{k+1}(x) d x\right] .
$$

The $\gamma$-entropy, say $H_{\gamma}(X)$, can be obtained as

where $\gamma>0, \gamma \neq 1$.

$$
H_{q}(X)=\frac{1}{\gamma-1} \log \left\{1-\left[\sum_{k=0}^{\infty} m_{k+1} \int_{-\infty}^{\infty} h_{k+1}(x) d x\right]\right\},
$$

\section{Maximum likelihood estimation}

Here, we determine the MLEs of the parameters of the new family of distributions from complete samples only. Let $X_{1}, \ldots, X_{n}$ be a random sample from the Po- $G$ family with parameters $\theta$ and $\xi$. Let $\Theta=\left(\theta, \xi^{\mathrm{T}}\right)^{T}$ be the $(p \times 1)$ parameter vector. Then, the loglikelihood function for $\Theta$, say $\ell=\ell(\Theta)$, is given by $\frac{\theta g(x ; \xi)}{e^{\theta}-1} \exp [\theta G(x ; \xi)]$

$$
\ell=n \log \theta-n \log \left(e^{\theta}-1\right)+\sum_{i=1}^{n} \log g\left(x_{i} ; \xi\right)+\theta \sum_{i=1}^{n} G\left(x_{i} ; \xi\right)
$$

Equation (8) can be maximized either directly by using the R (optim function), SAS (PROC NLMIXED) or Ox program (sub-routine MaxBFGS) or by solving the nonlinear likelihood equations obtained by differentiating (8).

The score vector components, say $\mathbf{U}(\Theta)=\frac{\partial \ell}{\partial \Theta}=\left(\frac{\partial \ell}{\partial \theta}, \frac{\partial \ell}{\xi_{k}}\right)^{\mathrm{T}}=\left(U_{\theta}, U_{\xi_{k}}\right)^{\mathrm{T}}$, are given by

$$
U_{\theta}=\frac{n}{\theta}-\frac{n e^{\theta}}{e^{\theta}-1}+\sum_{i=1}^{n} G\left(x_{i} ; \xi\right)
$$

and

$$
U_{\xi_{k}}=\sum_{i=1}^{n} \frac{1}{g\left(x_{i} ; \xi\right)} \frac{\partial g\left(x_{i} ; \xi\right)}{\partial \xi_{k}}+\theta \sum_{i=1}^{n} \frac{\partial G\left(x_{i} ; \xi\right)}{\partial \xi_{k}}
$$

Setting the nonlinear system of equations $U_{\theta}=U_{\xi_{k}}=\mathbf{0}$ and solving them simultaneously yields the MLE $\widehat{\Theta}=\left(\widehat{\theta}, \hat{\boldsymbol{\xi}}^{\mathrm{T}}\right)^{\mathrm{T}}$ of $\Theta=\left(\theta, \xi^{\mathrm{T}}\right)^{\mathrm{T}}$. These equations cannot be solved analytically and statistical software can be used to solve them numerically using iterative methods such as the Newton-Raphson type algorithms. For interval estimation of the parameters, we obtain the $p \times p$ observed information matrix $J(\Theta)=\left\{\frac{\partial^{2} \ell}{\partial r \partial s}\right\}$ (for $r, s=$ $\theta, \xi)$, whose elements can be computed numerically.

Under standard regularity conditions when $n \rightarrow \infty$, the distribution of $\widehat{\Theta}$ can be approximated by a multivariate normal $N_{p}\left(0, J(\widehat{\Theta})^{-1}\right)$ distribution to obtain confidence intervals for the parameters. Here, $J(\widehat{\Theta})$ is the total observed information matrix evaluated at $\widehat{\Theta}$. The method of the re-sampling bootstrap can be used for correcting the biases of the MLEs of the model parameters. Good interval estimates may also be obtained using the bootstrap percentile method. The elements of $J(\Theta)$ are given by

$$
U_{\theta \theta}=\frac{-n}{\theta^{2}}+\frac{(n-1) e^{2 \theta}+e^{\theta}}{e^{\theta}-1}, U_{\theta \xi_{k}}=\sum_{i=1}^{n} \frac{\partial G\left(x_{i} ; \xi\right)}{\partial \xi_{k}}
$$

and 


$$
U_{\xi_{k} \xi_{r}}=\sum_{i=1}^{n} \frac{1-g\left(x_{i} ; \xi\right)^{-1}}{g\left(x_{i} ; \xi\right)} \frac{\partial^{2} g\left(x_{i} ; \xi\right)}{\partial \xi_{k} \partial \xi_{r}}+\theta \sum_{i=1}^{n} \frac{\partial^{2} G\left(x_{i} ; \xi\right)}{\partial \xi_{k} \partial \xi_{r}} .
$$

\section{Simulation}

In this section for different combination of $\alpha, \beta$ and $\theta$, samples of sizes $n=$ $50,100,200,500$ and 1000 are generated from the PoW distribution. From the 1000 repetition we calculated the mean and the root mean square errors (RMSEs) of each parameters. Table 1 provides the results of the simulation results for two different combination of the PoW parameters. It can be clearly observed from these data that as sample size increases the mean square error decreases, it proves the consistency of the estimators.

Table 1: Empirical means and the RMSEs (in parentheses) of the PoW distribution

\begin{tabular}{|c|c|c|c|c|c|c|}
\hline$n$ & \multicolumn{5}{|c|}{ Parameters } \\
\hline & \multicolumn{2}{|c|}{$\alpha=1.5, \beta=2.5$ and $\theta=2$} & \multicolumn{2}{c|}{$\alpha=2.0, \beta=2.5$ and $\theta=3$} \\
\hline & $\hat{\alpha}$ & $\hat{\beta}$ & $\hat{\theta}$ & $\hat{\alpha}$ & $\hat{\beta}$ & $\hat{\theta}$ \\
\hline 50 & 1.593416 & 2.650682 & 2.423578 & 2.116847 & 2.740666 & 3.592813 \\
& $(0.5787654)$ & $(0.7052244)$ & $(3.514729)$ & $(0.6992011)$ & $(0.8705789)$ & $(4.416976)$ \\
\hline 100 & 1.529359 & 2.618779 & 2.032909 & 2.036667 & 2.685397 & 3.089807 \\
& $(0.305633)$ & $(0.5727127)$ & $(1.797346)$ & $(0.4455729)$ & $(0.7110352)$ & $(2.591586)$ \\
\hline 200 & 1.514847 & 2.588562 & 1.976926 & 2.001678 & 2.624106 & 2.924342 \\
& $(0.2472859)$ & $(0.4766343)$ & $(1.468686)$ & $(0.2926851)$ & $(0.5497098)$ & $(1.634355)$ \\
\hline 500 & 1.49699 & 2.571473 & 1.903862 & 1.978921 & 2.609259 & 2.844702 \\
& $(0.170683)$ & $(0.3780256)$ & $(1.090873)$ & $(0.2087809)$ & $(0.4347991)$ & $(1.179798)$ \\
\hline 1000 & 1.50043 & 2.535365 & 1.953882 & 1.986808 & 2.561902 & 2.907293 \\
& $(0.1309552)$ & $(0.2923231)$ & $(0.8294808)$ & $(0.1524578)$ & $(0.3134692)$ & $(0.8599232)$ \\
\hline
\end{tabular}

\section{Real data analysis}

In this section, we provide two applications to real data to illustrate the flexibility of the PoW, PoAW and PoBX models presented in Section 3. The goodness-of-fit statistics for these models are compared with other competitive models and the MLEs of the model parameters are determined. In order to compare the fitted models, we consider some goodness-of-fit measures including the Akaike information criterion $(A I C)$, consistent Akaike information criterion $(C A I C)$, Hannan-Quinn information criterion $(H Q I C)$, Bayesian information criterion $(B I C)$ and $-2 \hat{\ell}$, where $\hat{\ell}$ is the maximized log-likelihood. Further, we adopt the Anderson-Darling $\left(A^{*}\right)$ and Cramér-von Mises $\left(W^{*}\right)$ statistics in order to compare the fits of the two new models with other nested and non-nested models. The smaller these statistics are, the better the fit.

\subsection{The nicotine data}

The first data set refers to nicotine measurements, made from several brands of cigarettes in 1998, collected by the Federal Trade Commission which is an independent agency of the US government. The free form data set can be found at 
http://pw1.netcom.com/rdavis2/smoke.html. The site http://home.att.net/rda vis2/cigra.ht $\mathrm{ml}$ contains $\mathrm{n}=346$ observations.

We compare the fit of the PoW and PoAW distributions with those of other competitive models, namely: the additive Weibull (AW), beta Weibull (BW), transmuted Weibull Lomax (TWL), transmuted exponentiated generalized Weibull (TExGW), Kumaraswamy Weibull (Kw-W), New modified Weibull (NMW) and W distributions. The pdfs of these models are given in Appendix A.

\subsection{The gauge lengths data}

The second data set (gauge lengths of $20 \mathrm{~mm}$ ) (Kundu and Raqab, 2009) consists of 74 observations. For these data, we compare the fit of the PoW and PoBX distributions with those of the generalized transmuted Burr X (GTBX), exponentiated transmuted generalized Rayleigh (ETGR), TGR, GR and Rayleigh (R) models.

Tables 2 and 4 list the values of $-2 \hat{\ell}, A I C, C A I C, H Q I C, B I C, W^{*}$ and $A^{*}$, whereas the MLEs and their corresponding standard errors (in parentheses) of the model parameters are given in Tables 3 and 5.

In Table 2, we compare the fits of the PoW and PoAW models with the AW, BW, TWL, $\mathrm{Kw}-\mathrm{W}$, NMW, ETGR and Mc-W distributions. We note that the PoW and PoAW distributions have the lowest values for the $-2 \hat{\ell}, A I C, C A I C, H Q I C, B I C, W^{*}$ and $A^{*}$ statistics (for nicotine data) among all fitted models. So, the PoW and PoAW models could be chosen as the best models.

Table 2: Goodness-of-fit statistics for nicotine data

\begin{tabular}{|l|c|c|c|c|c|c|c|}
\hline \multicolumn{1}{|c|}{ Model } & $-2 \hat{\ell}$ & AIC & CAIC & HQIC & BIC & $W^{*}$ & $A^{*}$ \\
\hline PoW & 215.923 & 221.923 & 221.993 & 226.518 & 233.462 & 0.41905 & 2.31183 \\
\hline PoAW & 215.922 & 225.922 & 226.099 & 233.58 & 245.154 & 0.41527 & 2.28913 \\
\hline BW & 225.173 & 233.173 & 233.29 & 239.30 & 248.559 & 0.49664 & 2.89774 \\
\hline AW & 226.581 & 234.581 & 234.698 & 240.707 & 249.966 & 0.55222 & 3.17512 \\
\hline TWL & 225.832 & 235.832 & 236.009 & 243.491 & 255.064 & 0.50049 & 2.92291 \\
\hline TExGW & 226.317 & 236.317 & 236.494 & 243.976 & 255.55 & 0.5205 & 3.02977 \\
\hline KW-W & 226.184 & 234.184 & 234.302 & 240.311 & 249.57 & 0.5325 & 3.08454 \\
\hline NMW & 225.284 & 235.284 & 235.461 & 242.943 & 254.517 & 0.49269 & 2.87829 \\
\hline W & 226.581 & 230.581 & 230.616 & 233.644 & 238.274 & 0.55744 & 3.20719 \\
\hline
\end{tabular}

In Table 4, we compare the fits of the PoW and PoBX models with the GTBX, ETGR, TGR, GR and R models. The figures in this table reveal that the PoW and PoBX models have the lowest values for $-2 \widehat{\ell}, A I C, C A I C, H Q I C, B I C, W^{*}$ and $A^{*}$ statistics (for gauge lengths data) among all fitted models. So, the PoW and PoBX distributions can be chosen as the best models. So, we prove that these new distributions can be better models than other competitive lifetime models. 
The Poisson-G Family of Distributions with Applications

Table 3 : MLEs and their standard errors (in parentheses) for nicotine data

\begin{tabular}{|l|c|c|c|c|c|}
\hline Model & \multicolumn{5}{|c|}{ Estimates } \\
\hline PoW & $\hat{\alpha}=1.4575$ & $\hat{\beta}=1.9347$ & $\hat{\theta}=2.8271$ & & \\
& $(0.134)$ & $(0.194)$ & $(0.744)$ & & \\
\hline PoAW & $\hat{\alpha}=2.0673$ & $\hat{\beta}=0.6039$ & $\hat{\gamma}=0.0053$ & $\hat{\delta}=1.9391$ & $\hat{\theta}=2.828$ \\
& $(0.232)$ & $(0.015)$ & $\left(2.917 \times 10^{-3}\right)$ & $(0.196)$ & $(0.745)$ \\
\hline BW & $\hat{\alpha}=0.6686$ & $\hat{\beta}=3.1645$ & $\hat{a}=0.7784$ & $\hat{b}=3.0922$ & \\
& $(0.578)$ & $(0.426)$ & $(0.163)$ & $(8.174)$ & \\
\hline AW & $\hat{\alpha}=1.135$ & $\hat{\beta}=0.3084$ & $\hat{\gamma}=0.0002$ & $\hat{\delta}=2.7219$ & \\
& $(0.062)$ & $(0.1)$ & $\left(1.369 \times 10^{-3}\right)$ & $(0.114)$ & \\
\hline TWL & $\hat{\alpha}=7.8903$ & $\hat{\beta}=17.1065$ & $\hat{a}=3.8175$ & $\hat{b}=2.3499$ & $\hat{\lambda}=0.16$ \\
& $(15.497)$ & $(44.562)$ & $(4.1843)$ & $(0.309)$ & $(0.214)$ \\
\hline TExGW & $\hat{\alpha}=1.0589$ & $\hat{\beta}=3.3201$ & $\hat{\lambda}=0.2257$ & $\hat{a}=0.6338$ & $\hat{b}=0.7665$ \\
& $(4.268)$ & $(0.279)$ & $(0.23)$ & $(8.482)$ & $(0.13)$ \\
\hline KW-W & $\hat{\alpha}=0.6157$ & $\hat{\beta}=3.1187$ & $\hat{a}=0.8395$ & $\hat{b}=3.7931$ & \\
& $(0.392)$ & $(0.698)$ & $(0.233)$ & $(6.921)$ & \\
\hline NMW & $\hat{\alpha}=0.0012$ & $\hat{\beta}=2.3518$ & $\hat{\gamma}=0.7453$ & $\hat{\delta}=0.3956$ & $\hat{\theta}=2.083$ \\
& $(0.036)$ & $(0.337)$ & $(0.276)$ & $(0.344)$ & $(0.584)$ \\
\hline W & $\hat{\alpha}=1.0477$ & $\hat{\beta}=2.7208$ & & & \\
& $(0.022)$ & $(0.114)$ & & & \\
\hline
\end{tabular}

The histogram and the estimated densities for nicotine and gauge lengths data are displayed in Figure 4. QQ-plots for the best fitted distributions are shown in Figure 5. From these graphs it is evident that the PoW, PoAW and PoBX distributions best describe both data sets.
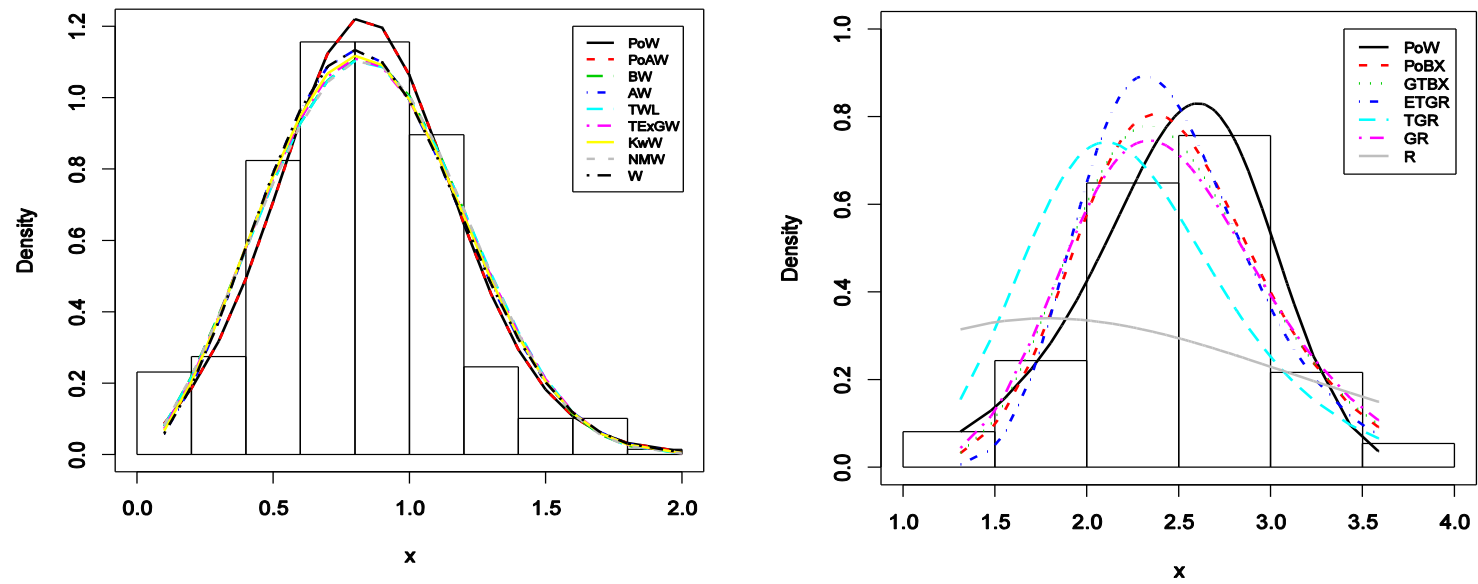

Figure 4: Fitted pdfs for nicotine data (left panel) and for gauge lengths data (right panel)

Table 4 : Goodness-of-fit statistics for gauge lengths data

\begin{tabular}{|l|c|c|c|c|c|c|c|}
\hline Model & $-2 \hat{\ell}$ & $A I C$ & CAIC & HQIC & $B I C$ & $W^{*}$ & $A^{*}$ \\
\hline PoW & 103.45 & 109.45 & 109.793 & 112.207 & 116.362 & 0.0287 & 0.25174 \\
\hline PoBX & 106.659 & 112.659 & 113.001 & 115.416 & 119.571 & 0.09016 & 0.57835 \\
\hline GTBX & 108.055 & 118.055 & 118.937 & 122.65 & 129.575 & 0.10458 & 0.68807 \\
\hline ETGR & 113.4 & 121.352 & 121.9 & 125.029 & 130.6 & 0.20714 & 1.3407 \\
\hline TGR & 123.61 & 129.61 & 129.95 & 132.376 & 136.5 & 0.16923 & 1.28629 \\
\hline GR & 135.202 & 139.202 & 139.371 & 141.041 & 143.811 & 0.13403 & 0.86836 \\
\hline R & 188.302 & 190.302 & 190.375 & 191.221 & 192.606 & 1.77111 & 32.95987 \\
\hline
\end{tabular}


Table 5 : MLEs and their standard errors (in parentheses) for gauge lengths data

\begin{tabular}{|c|c|c|c|c|c|}
\hline Model & & & Estimate & & \\
\hline PoW & $\begin{array}{c}\hat{\alpha}=0.405 \\
(0.054)\end{array}$ & $\begin{array}{c}\hat{\beta}=4.8081 \\
(1.424)\end{array}$ & $\begin{array}{c}\hat{\theta}=1.4149 \\
(2.28)\end{array}$ & & \\
\hline PoBX & $\begin{array}{c}\hat{\alpha}=0.6444 \\
(0.686)\end{array}$ & $\begin{array}{c}\hat{\beta}=0.681 \\
(0.029)\end{array}$ & $\begin{array}{c}\hat{\theta}=17.0518 \\
(15.441)\end{array}$ & & \\
\hline GTBX & $\begin{array}{c}\hat{\alpha}=3.4900 \\
(2.084)\end{array}$ & $\begin{array}{c}\hat{\beta}=0.6615 \\
(0.12)\end{array}$ & $\begin{array}{c}\hat{a}=2.5190 \\
(1.503)\end{array}$ & $\begin{array}{c}\hat{b}=0.0161 \\
(0.428)\end{array}$ & $\begin{array}{c}\hat{\lambda}=0.0019 \\
(0.048)\end{array}$ \\
\hline ETGR & $\begin{array}{c}\hat{\alpha}=2.1214 \\
(0.315)\end{array}$ & $\begin{array}{c}\hat{\beta}=0.6985 \\
(0.040)\end{array}$ & $\begin{array}{c}\hat{\lambda}=0.3201 \\
(0.228)\end{array}$ & $\begin{array}{c}\hat{\delta}=7.790 \\
(1.727)\end{array}$ & \\
\hline TGR & $\begin{array}{c}\hat{\alpha}=5.5052 \\
(0.776)\end{array}$ & $\begin{array}{c}\hat{\beta}=0.6245 \\
(0.017)\end{array}$ & $\begin{array}{c}\hat{\lambda}=0.3599 \\
(0.253)\end{array}$ & & \\
\hline GR & $\begin{array}{c}\hat{\alpha}=7.784 \\
(1.625)\end{array}$ & $\begin{array}{c}\hat{\beta}=0.6445 \\
(0.024)\end{array}$ & & & \\
\hline $\mathrm{R}$ & $\begin{array}{c}\hat{\beta}=0.3962 \\
(0.023)\end{array}$ & & & & \\
\hline
\end{tabular}

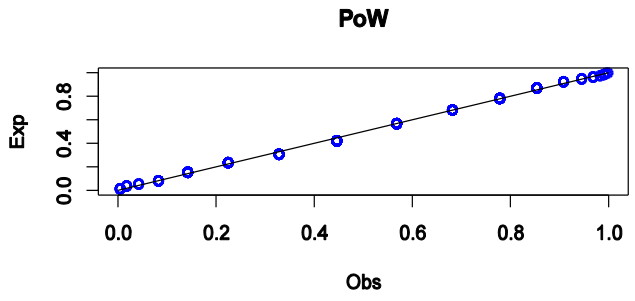

POAW

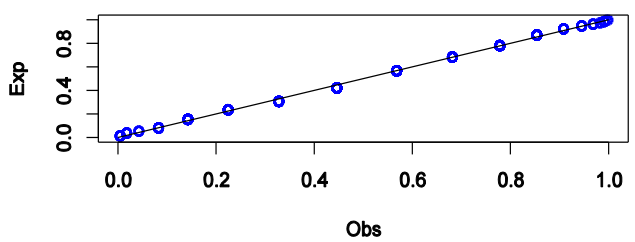

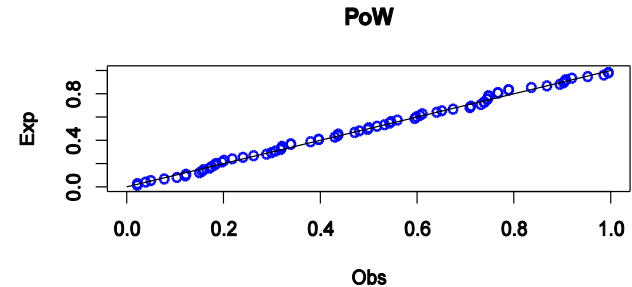

PoBX

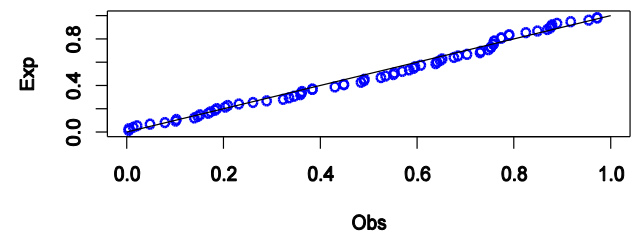

Figure 5: QQ plots for the PoW and PoAW models (left panel) and PoW and PoBX models (right panel)

\section{Conclusions}

The idea of generating new extended models from classic ones has been of great interest among researchers in the past decade. We present a new Poisson-G (Po-G) family of distributions. We provide some mathematical properties of the new family including explicit expansions for the ordinary and incomplete moments, quantile and generating functions and entropies. The maximum likelihood estimation of the model parameters is investigated and the observed information matrix is determined. By means of two real data sets, we verify that special cases of the Po- $G$ family can provide better fits than other models generated from well-known families.

\section{Appendix A:}

The pdfs of the fitted models are given (for $x>0$ ): 
- The AW density (Xie and Lai, 1995) given by

$$
f(x)=\left(\alpha \delta x^{\delta-1}+\gamma \beta x^{\beta-1}\right) e^{-\alpha x^{\delta}-\gamma x^{\beta}},
$$

where $\alpha>0, \beta>0, \gamma>0$ and $\delta>0$.

- The BW density (Lee et al., 2007) given by

$$
f(x)=\frac{\beta \alpha^{\beta}}{B(a, b)} x^{\beta-1} e^{-b(\alpha x)^{\beta}}\left[1-e^{-(\alpha x)^{\beta}}\right]^{a-1},
$$

where $\alpha>0, \beta>0, a>0$ and $b>0$.

- The TWL density (Afify et al., 2015) given by

$$
\begin{aligned}
& f(x)=\frac{a b \alpha}{\beta}\left(1+\frac{x}{\beta}\right)^{b \alpha-1} \exp \left\{-a\left[\left(1+\frac{x}{\beta}\right)^{\alpha}-1\right]^{b}\right\} \\
& \times\left[1-\left(1+\frac{x}{\beta}\right)^{-\alpha}\right]^{b-1}\left\{1-\lambda+2 \lambda e^{-a\left[\left(1+\frac{x}{\beta}\right)^{\alpha}-1\right]^{b}}\right\},
\end{aligned}
$$

where $\alpha>0, \beta>0, a>0, b>0$ and $|\lambda| \leq 1$.

- The TExGW density (Yousof et al., 2015) given by

$$
f(x)=a b \beta \alpha^{\beta} x^{\beta-1} e^{-a(\alpha x)^{\beta}}\left[1-e^{-a(\alpha x)^{\beta}}\right]^{b-1}\left\{1+\lambda-2 \lambda\left[1-e^{-a(\alpha x)^{\beta}}\right]^{b}\right\},
$$

where $\alpha>0, \beta>0, a>0, b>0$ and $|\lambda| \leq 1$.

- The Kw-W density (Cordeiro et al., 2010) given by

$$
f(x)=a b \beta \alpha^{\beta} x^{\beta-1} e^{-(\alpha x)^{\beta}}\left[1-e^{-(\alpha x)^{\beta}}\right]^{a-1}\left\{1-\left[1-e^{-(\alpha x)^{\beta}}\right]^{a}\right\}^{b-1},
$$

where $\alpha>0, \beta>0, a>0$ and $b>0$.

- The NMW density (Almalki and Yuan, 2013) given by

$$
f(x)=\left(\alpha \theta x^{\theta-1}+\gamma(\beta+\delta x) x^{\beta-1} e^{\delta x}\right) e^{-\left(\alpha x^{\theta}+\gamma x^{\beta}\right) e^{\delta x}}
$$

where $\alpha>0, \beta>0, \gamma>0, \delta>0$ and $\theta>0$.

- The GTBX density (Nofal et al., 2017) given by

$$
f(x)=2 \alpha \beta^{2} x e^{-(\beta x)^{2}}\left[1-e^{-(\beta x)^{2}}\right]^{\alpha a-1} a\left\{(1+\lambda)-\lambda(a+b)\left[1-e^{-(\beta x)^{2}}\right]^{\alpha b}\right\},
$$

where $\alpha>0, \beta>0, a>0, b>0$ and $|\lambda| \leq 1$.

- The ETGR density (Afify et al., 2015) given by

$$
\begin{gathered}
f(x)=2 \alpha \delta \beta^{2} x e^{-(\beta x)^{2}}\left[1-e^{-(\beta x)^{2}}\right]^{\alpha \delta-1} \\
\times\left\{1+\lambda-2 \lambda\left[1-e^{-(\beta x)^{2}}\right]^{\alpha}\right\}\left\{1+\lambda-\lambda\left[1-e^{-(\beta x)^{2}}\right]^{\alpha}\right\}^{\delta-1}
\end{gathered}
$$

where $\alpha>0, \beta>0, \delta>0$ and $|\lambda| \leq 1$.

\section{References}

1. Afify, A. Z., Nofal, Z. M. and Ebraheim, A. N. (2015). Exponentiated transmuted generalized Rayleigh distribution: a new four parameter Rayleigh distribution. Pak. J.

Stat. Oper. Res., 11, 115-134. 
2. Afify, A. Z., Nofal, Z. M., Yousof, H. M., El Gebaly, Y. M. and Butt, N. S. (2015). The transmuted Weibull Lomax distribution: properties and application, Pak. J. Stat. Oper. Res., 11, 135-152.

3. Alexander, C., Cordeiro, G. M., Ortega, E. M. M. and Sarabia, J. M. (2012). Generalized beta-generated distributions. Computational Statistics and Data Analysis, 56, 1880-1897.

4. Almalki, S. J. and Yuan, J. (2013). A new modified Weibull distribution. Reliability Engineering and System Safety, 111, 164-170.

5. Alzaatreh, A., Lee, C. and Famoye, F. (2013). A new method for generating families of continuous distributions. Metron, 71, 63-79.

6. Cordeiro, G. M. and de Castro, M. (2011). A new family of generalized distributions. J. Stat. Comput. Simul., 81, 883-893.

7. Cordeiro, G. M., Ortega, E. M., and Nadarajah, S. (2010). The Kumaraswamy Weibull distribution with application to failure data. Journal of the Franklin Institute, 347, 1399-1429.

8. Eugene, N., Lee, C. and Famoye, F. (2002). Beta-normal distribution and its applications. Commun. Stat. Theory Methods, 31, 497-512.

9. Kundu, D. and Raqab, M. Z. (2009). Estimation of $R=P(Y<X)$ for threeparameter Weibull distribution. Statistics and Probability Letters, 79, 1839-1846.

10. Lee, C., Famoye, F., and Olumolade, O. (2007). Beta-Weibull distribution: some properties and applications to censored data. Journal of modern applied statistical methods, $6,17$.

11. Mahmoudi, E. and Jafari, A. A. (2012). Generalized exponential-power series distributions. Computational Statistics and Data Analysis, 56, 4047-4066.

12. Nofal, Z. M., Afify, A. Z., Yousof, H. M. and Cordeiro, G. (2017). The generalized transmuted-G family of distributions. Communications in Statistics-Theory and Methods, 46, 4119-4136.

13. Shaw, W. T. and Buckley, I. R. C. (2007). The alchemy of probability distributions: beyond Gram-Charlier expansions and a skew-kurtotic-normal distribution from a rank transmutation map. arXiv preprint arXiv:0901.0434.

14. Xie, M. and Lai, C. D. (1995). Reliability analysis using an additive Weibull model with bathtub-shaped failure rate function. Reliability Engineering and System Safety, 52, 87-93.

15. Yousof, H. M., Afify, A. Z., Alizadeh, M., Butt, N. S., Hamedani, G. G. and Ali, M. M. (2015). The transmuted exponentiated generalized-G family of distributions. Pakistan Journal of Statistics and Operations Research, 11, 441-464. 